\title{
Extensive high-precision studies of proton deuteron breakup reactions at COSY
}

\section{P. Thörngren Engblom; S. Bertelli, M. Contalbrigo, P. Lenisa}

University of Ferrara and INFN, 44100 Ferrara, Italy

E-mail: thorngrenefysik.su.se, bertelliefe.infn.it,

mcontalbefe.infn.it,lenisa@fe.infn.it

D. Chiladze, A. Kacharava, B. Lorentz, A. Nass, D. Oellers, F. Rathmann, R. Schleichert, H. Ströher, C. Weidemann

Institut für Kernphysik and Jülich Centre for Hadron Physics, Forschungszentrum Jülich, 52425

Jülich, Germany

E-mail: d.chiladze@fz-juelich.de, a.kacharava@fz-juelich.de,

b.lorentz@fz-juelich.de, a.nass@fz-juelich.de,

di.oellersafz-juelich.de, f.rathmannefz-juelich.de,

r.schleichertefz-juelich.de, h.stroeherefz-juelich.de,

c.weidemannefz-juelich.de

\section{N. Lomidze, M. Tabidze}

High Energy Physics Institute, Tbilisi State University, 0186 Tbilisi, Georgia

E-mail: n. lomidze@hepi.edu.ge, m.tabidze@hepi.edu.ge

\section{G. Macharashvili, S. Merzlyakov}

Laboratory of Nuclear Problems, JINR, RU-141980 Dubna, Russia

E-mail: gogi@nusun.jinr.ru, simenusun.jinr.ru

\section{S. Barsov, S. Mikirtytchiants}

Petersburg Nuclear Physics Institute, RU-188350 Gatchina, Russia

E-mail: barsov@pnpi.spb.ru, s.mikirtytchiants@fz-juelich.de

We plan to measure the spin dependence of proton deuteron breakup at 30 and $49 \mathrm{MeV}$ proton beam energy where previous measurements are few and limited. The physics objective is to test the predictive power of the chiral effective field theory in the three nucleon continuum by measuring analyzing powers and double spin observables with high precision over large areas of phase space at relevant energies for the theoretical interpretation. The experiment will be done at a newly installed and commissioned low- $\beta$ section and interaction point in the COSY ring utilizing the PAX Multipurpose Detection System that is presently in the design stage.

PACS: $21.45 .-\mathrm{V}, 21.45 . \mathrm{Ff}, 24.70 .+\mathrm{s}, 29.85 . \mathrm{Fj}$

8th International Conference on Nuclear Physics at Storage Rings-Stori11,

October 9-14, 2011

Laboratori Nazionali di Frascati dell'INFN, Italy

\footnotetext{
* Speaker.

$\dagger$ on behalf of the PAX and ANKE Collaborations

$\ddagger$ Joint affiliation: KTH Royal Institute of Technology, AlbaNova University Center, SE - 10691 Stockholm, Sweden
} 


\section{Introduction}

Presently the spin structure of the three nucleon continuum exhibits disparate results when comparing experimental data to theoretical predictions based on two-nucleon potentials either with or without three nucleon interactions. The inclusion of three-nucleon forces in the calculations does not consistently improve the agreement between data and theory $[1,2,3,4,5,6]$. A recent review on the current status of the research on the nature of three-nucleon forces (3NF) can be found in [7] considering aspects from both experiment and theory.

The rich kinematical region in proton deuteron breakup reactions at low to intermediate energy offers a versatile laboratory for the chiral effective field theory (EFT), the modern theory of nuclear forces in the low-energy regime; see [8, 9] and references therein. In the framework of chiral EFT nucleon-nucleon potentials have been developed to next-to-next-to-next-to leading order (N3LO) reproducing two-nucleon observables with high accuracy [10,11]. Three-nucleon forces (3NF) have been analyzed up to next-to-next-to-leading order (N2LO) with persistent discrepancies and inconclusive results as to specifically how the presence of a third nucleon affect the interaction in few-nucleon systems. In order to remedy this situation both the long-range [12] and short-range contributions [13] to the $3 \mathrm{~N}$ forces were derived up to N3LO. Thus the calculations of two-, threeand four-nucleon forces at this order in the chiral expansion (without explicit $\Delta(1232)$ degrees of freedom) is completed and the implementation into the theoretical calculations for scattering observables at low to intermediate energy of three nucleon configurations is in progress [14].

Precise measurements of the majority of the spin observables over large areas of phase space would provide a data base for the characterization of the non-vanishing contributions from the chiral 3NF appearing at third and fourth order. To this aim we plan an experiment at the COSY cooler synchrotron and storage ring, measuring proton deuteron breakup reactions at proton beam energies from 30 to $50 \mathrm{MeV}$ where few previous data exist. This is also an ideal energy range for testing the predictive power of chiral EFT. In addition the only two low energy constants (LECs) needed for both N2LO and N3LO, $D$ and $E$, could be independently determined from a multitude of scattering spin observables ${ }^{1}$.

The experiment will be carried out in one of the straight sections in the COSY ring, where a low $\beta$ section has been installed for the commissioning of the spin filtering experiment [15] with the objective of polarizing a stored antiproton beam in situ that is proposed by the PAX Collaboration to be done at the AD ring at CERN [16]. The experimental details and the plans for a PAX Multipurpose Detection System, barrel shaped with layers of silicon double sided strip detectors, are given in [17]. A precursor $p d$ breakup experiment using vertically polarized beam, an unpolarized deuterium target and the ANKE silicon telescopes is currently being analyzed.

\section{Observables and Theoretical Predictions from Chiral EFT}

The formalism for polarization in nuclear reactions was worked out in detail by G.G. Ohlsen in the seventies [18]. The general expression for the double polarized proton deuteron breakup reaction can be found in [19]. Here is shown in table form the independent observables categorized

\footnotetext{
${ }^{1}$ These LECs are currently deduced from the ${ }^{3} H$ binding energy and the $n d$ doublet scattering length, the properties of light nuclei, the triton $\beta$ decay or the ${ }^{4} \mathrm{He}$ binding energy.
} 
by the spin alignment directions of the proton beam and the deuterium target required to access the specified observable, see Table [1].

\subsection{Grid studies}

In order to facilitate a theoretical investigation for the optimization of the measurements of all the spin observables, grids were created based on chiral EFT at N2LO, one for each observable ${ }^{2}$, with the theoretical predictions as functions of the five kinematically independent parameters, $p$, $\theta_{p}, \theta_{q}, \phi_{p}$ and $\phi_{q}$; where $p$ and $q$ are the jacobi momenta in the center-of-mass. The grids are thus large matrices spanning the available phase space. Using the sampling method, thoroughly explained in [20] and applied in [3,21], enables a systematic study of any three-particle final state. For the current study the sampled events are isotropically generated in a phase space simulation of $p d$ breakup. Then multidimensional linear interpolation is used to retrieve the theoretical prediction for each event. In the following figures a few examples of the application are given, all for a proton beam energy of $49 \mathrm{MeV}$. Some aspects are displayed of the tensor analyzing power $A_{x z}$ in Figs. 1 4, the tensor-vector correlation coefficient $C_{y y, y}$ in Figs. $5-7$ and the vector-vector spin correlation $C_{x, x}$ in Figs. 8 and 9.

The foreseen final result for the planned $p d$ breakup experiment is a compilation of the data from all measured observables leading to an overall evaluation of the degree of agreement between experiment and theory.

\section{Summary}

Measurements of proton deuteron breakup at energies between 30 and $49 \mathrm{MeV}$ are planned at the COSY accelerator and storage ring, motivated by the development of the chiral EFT for describing low-energy phenomena in hadron physics. Using a new experimental approach the aim is to obtain conclusive constraints for the chiral $3 \mathrm{NFs}$, and to form a data base of analyzing powers and spin correlation coefficients at low energy thus contributing to a fuller understanding of fewnucleon interactions.

\section{Acknowledgements}

We would like to express our gratitude to Prof. E. Epelbaum and Dr. A. Nogga who provide the chiral EFT framework, the chiral $3 \mathrm{~N}$ forces, and their implementation in producing the theory grids.

This publication has been supported by the European Commission under the 7th Framework Programme through the 'Research Infrastructures' action of the 'Capacities' Programme. Call: FP7-INFRASTRUCTURES-2008-1, Grant Agreement N. 227431. The work has been carried out within Work Packages 13 and 25 of the project Study of Strongly Interacting Matter (acronym: Hadronphysics2). We would also like to acknowledge the support by the Swedish Research Council, grant nos. 2009-2240 and 2010-5135.

\footnotetext{
${ }^{2}$ The theory grids were created by A. Nogga
} 


\begin{tabular}{|l|c|c|c|c|c|c|c|c|}
\hline PolObs & $p \mathrm{U} d \mathrm{U}$ & $p \mathrm{U} d \mathrm{~S}$ & $p \mathrm{U} d \mathrm{~A}$ & $p \mathrm{~A} d \mathrm{U}$ & $p \mathrm{~A} d \mathrm{~S}$ & $p \mathrm{~A} d \mathrm{~A}$ & $p \mathrm{U} d \mathrm{AU}$ & $p \mathrm{U} d \mathrm{AS}$ \\
\hline \hline$A_{y}(p)$ & $\mathrm{X}$ & $\mathrm{X}$ & $\mathrm{X}$ & & & & $\mathrm{X}$ & $\mathrm{X}$ \\
\hline $\mathbf{A}_{\mathbf{z}}(\mathbf{p})$ & & & & $\mathrm{X}$ & $\mathrm{X}$ & $\mathrm{X}$ & $p \mathrm{~A} d \mathrm{AU}$ & $p \mathrm{~A} d \mathrm{AS}$ \\
\hline$A_{y}(d)$ & $\mathrm{X}$ & $\mathrm{X}$ & & $\mathrm{X}$ & $\mathrm{X}$ & & $\mathrm{X}$ & $\mathrm{X}$ \\
\hline $\mathbf{A}_{\mathbf{z}}(\mathbf{d})$ & & & $\mathrm{X}$ & & & $\mathrm{X}$ & $\mathrm{X}$ & $\mathrm{X}$ \\
\hline$A_{x x}-A_{y y}$ & $\mathrm{X}$ & $\mathrm{X}$ & & $\mathrm{X}$ & $\mathrm{X}$ & & $\mathrm{X}$ & $\mathrm{X}$ \\
\hline$A_{z z}$ & $\mathrm{X}$ & $\mathrm{X}$ & $\mathrm{X}$ & $\mathrm{X}$ & $\mathrm{X}$ & $\mathrm{X}$ & $\mathrm{X}$ & $\mathrm{X}$ \\
\hline$A_{x z}$ & & & & & & & $\mathrm{X}$ & $\mathrm{X}$ \\
\hline$C_{x, x}+C_{y, y}$ & $\mathrm{X}$ & & & & & & $\mathrm{X}$ & \\
\hline$C_{x, x}-C_{y, y}$ & $\mathrm{X}$ & $\mathrm{X}$ & & & & & $\mathrm{X}$ & $\mathrm{X}$ \\
\hline $\mathbf{C}_{\mathbf{y}, \mathbf{x}}-\mathbf{C}_{\mathbf{x}, \mathbf{y}}$ & & $\mathrm{X}$ & & & & & & $\mathrm{X}$ \\
\hline$C_{x, z}$ & & & & $\mathrm{X}$ & $\mathrm{X}$ & & $p \mathrm{~A} d \mathrm{AU}$ & $p \mathrm{~A} d \mathrm{AS}$ \\
\hline$C_{z, x}$ & & & $\mathrm{X}$ & & & & $\mathrm{X}$ & $\mathrm{X}$ \\
\hline$C_{z, z}$ & & & & & & $\mathrm{X}$ & $p \mathrm{~A} d \mathrm{AU}$ & $p \mathrm{~A} d \mathrm{AS}$ \\
\hline$C_{x x, y}-C_{y y, y}$ & $\mathrm{X}$ & $\mathrm{X}$ & & & & & $\mathrm{X}$ & $\mathrm{X}$ \\
\hline $\mathbf{C}_{\mathbf{x z}, \mathbf{x}}+\mathbf{C}_{\mathbf{y z}, \mathbf{y}}$ & & & & & & $\mathrm{X}$ & $\mathrm{X}$ & \\
\hline $\mathbf{C}_{\mathbf{z z}, \mathbf{z}}$ & & & & $\mathrm{X}$ & $\mathrm{X}$ & $\mathrm{X}$ & $p \mathrm{~A} d \mathrm{AU}$ & $p \mathrm{~A} d \mathrm{AS}$ \\
\hline$C_{z z, y}$ & $\mathrm{X}$ & $\mathrm{X}$ & $\mathrm{X}$ & & & & $\mathrm{X}$ & $\mathrm{X}$ \\
\hline$C_{x y, x}$ & $\mathrm{X}$ & $\mathrm{X}$ & & & & & $\mathrm{X}$ & $\mathrm{X}$ \\
\hline$C_{x z, y}$ & & & & & & & $\mathrm{X}$ & $\mathrm{X}$ \\
\hline$C_{y z, x}$ & & & & & & & $\mathrm{X}$ & $\mathrm{X}$ \\
\hline$C_{x y, z}$ & & & & $\mathrm{X}$ & $\mathrm{X}$ & & $p \mathrm{~A} d \mathrm{AU}$ & $p \mathrm{~A} d \mathrm{AS}$ \\
\hline$C_{y z, z}$ & & & & & & & $p \mathrm{~A} d \mathrm{AU}$ & $p \mathrm{~A} d \mathrm{AS}$ \\
\hline \hline
\end{tabular}

Table 1: Tabulated here are the 7 analyzing powers and 15 spin correlation observables possible in proton deuteron breakup showing the required polarization alignment directions of beam and target and some combinations thereof. For $p$ (proton) and $d$ (deuteron); U means alignment up (vertical), $\mathrm{S}$ is sideways (parallell to the $\mathrm{x}$-axis) and $\mathrm{A}$ is along the beam direction (longitudinal). The last two columns refer to the situation when the deuteron target spin alignment axis is at 45 degrees which can be accomplished by running current through two target guide field coils simultaneously. With the longitudinal $( \pm z)$ and vertical $( \pm y)$ guide field coils on, denoted $d \mathrm{AU}$, and switched in \pm polarity, four directions are achieved. Another four alignments are obtained with the longitudinal and sideways $( \pm x)$ combinations, denoted $d \mathrm{AS}$. There are five observables (here marked in bold font) that are parity forbidden in elastic scattering and goes to zero in breakup reactions in coplanar kinematical configurations. In the last two columns also a few observables are included requiring longitudinally polarized beam in addition to a target spin alignment at 45 degrees. E.g. the tensor-vector correlation coefficient $C_{y z, z}$ is accessible only using longitudinally polarized beam and diagonal target spin alignment. 

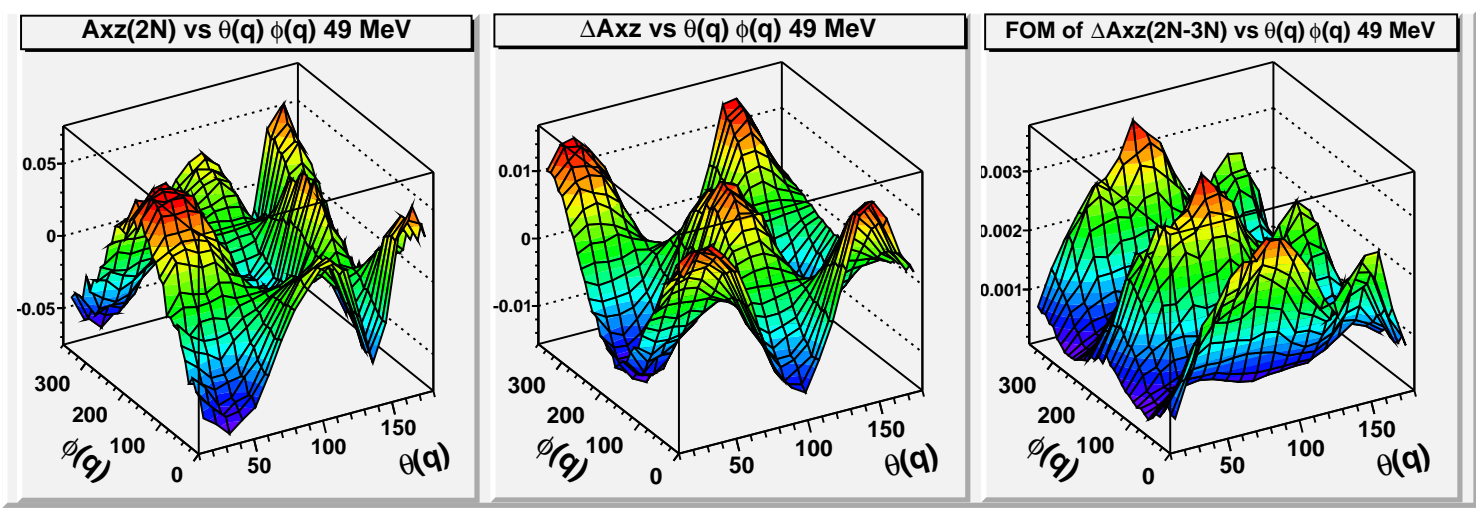

Figure 1: The tensor analyzing power $A_{x z}(2 \mathrm{~N})($ left $)$, the difference between the $2 \mathrm{~N}$ and $3 \mathrm{~N}$ predictions (middle), the figure-of-merit (right) as function of $\theta_{q}$ and $\phi_{q}$.
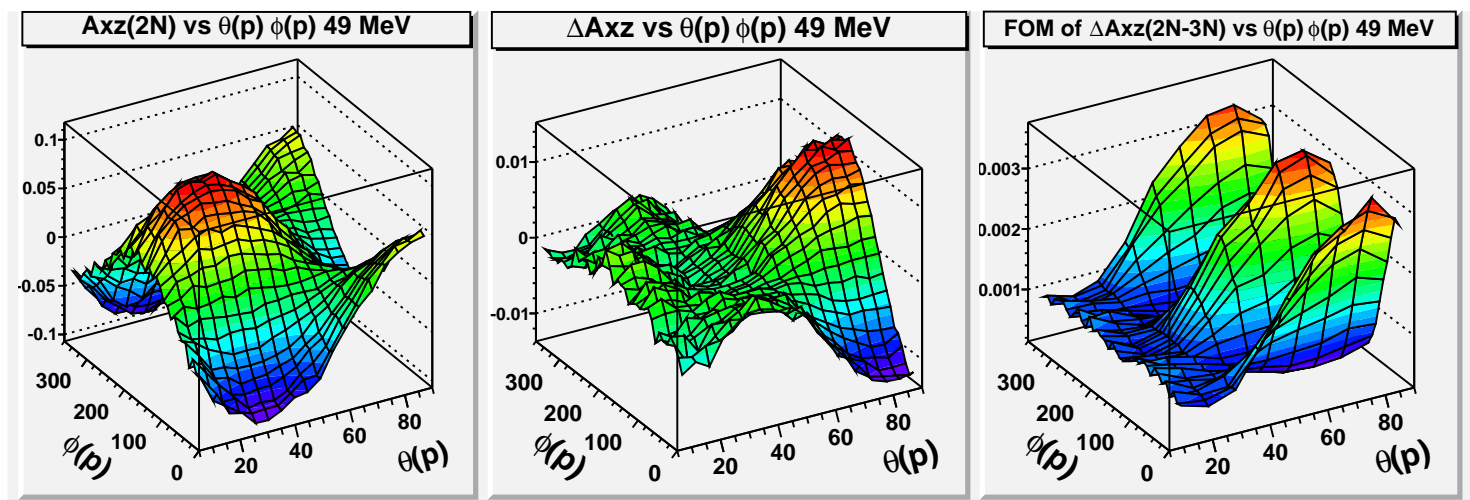

Figure 2: The tensor analyzing power $A_{x z}(2 \mathrm{~N})($ left $)$, the difference between the $2 \mathrm{~N}$ and $3 \mathrm{~N}$ predictions (middle), the figure-of-merit (right) as function of $\theta_{p}$ and $\phi_{p}$.

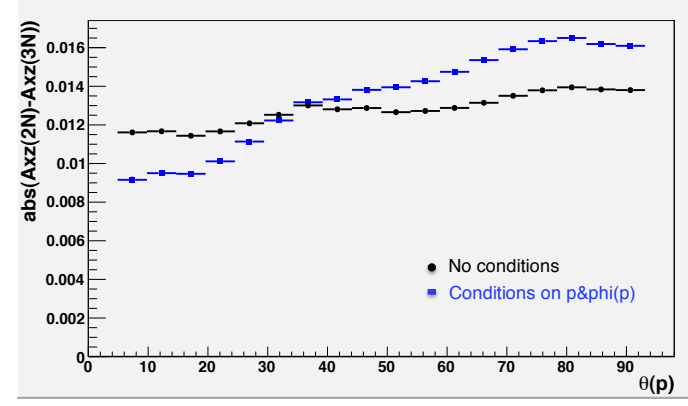

Figure 3: The absolute magnitude of the difference of the tensor analyzing power $A_{x z}(2 N)-A_{x z}(3 N)$ as function of $\theta_{p}$ in a profile histogram. The dots (black) represent no selection but integration over all independent variables. The squares (blue online) represent conditions set on $p$ and $\phi_{p}$. For $\theta_{p}>45^{\circ}$ a larger difference is observed between $2 \mathrm{~N}$ and $3 \mathrm{~N}$ calculations.

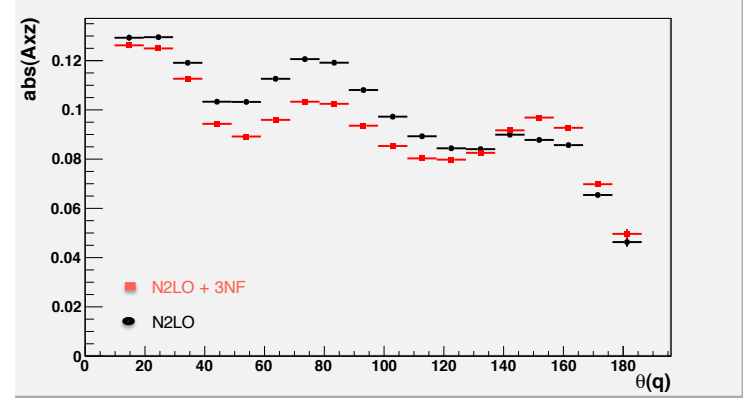

Figure 4: The absolute magnitude of the tensor analyzing power $A_{x z}$ as function of $\theta_{q}$ in a profile histogram. The dots (black) represents predictions according the $2 \mathrm{~N}$ calculation, the squares (red online) includes $3 \mathrm{~N}$ interactions. Cuts have been made on $p, \theta_{p}, \phi_{p}$ and $\phi_{q}$ in order to visualize one part of phase space where significant deviations might appear, roughly 0.02 between 60 and 90 degrees. 

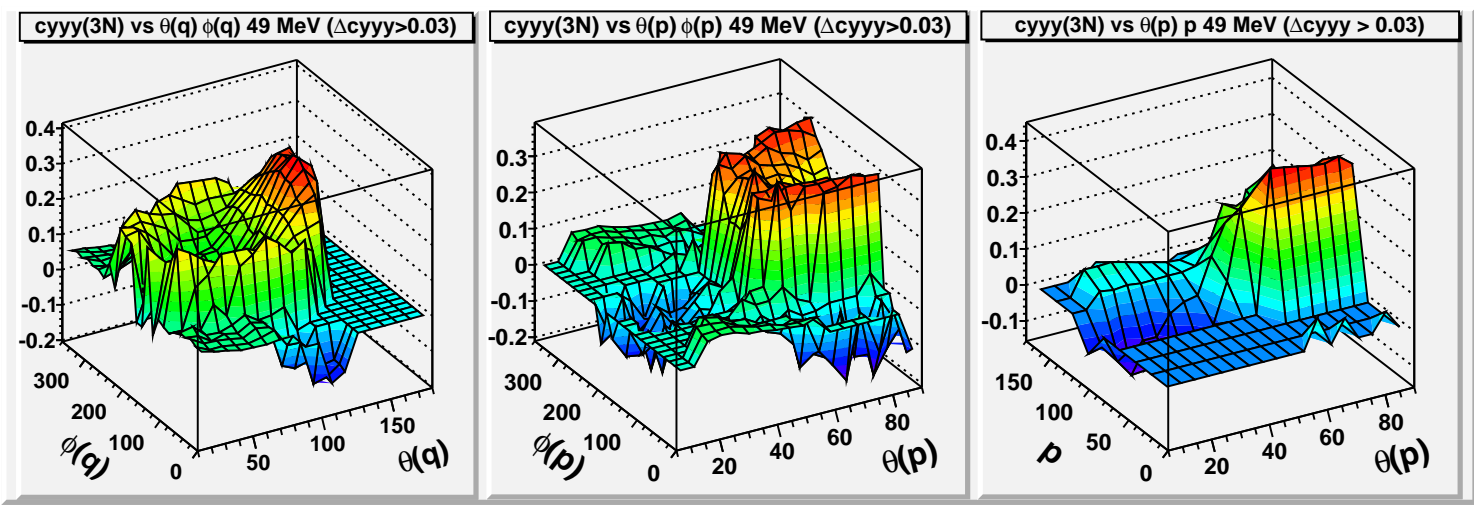

Figure 5: The tensor-vector correlation coefficient $C_{y y, y}(2 \mathrm{~N})$ as function of $\theta_{q}$ and $\phi_{q}$ (left), $\theta_{p}$ and $\phi_{p}$ (middle), $\theta_{p}$ and $p$ (right), depicted in two dimensional profile histograms under the condition that the absolute difference between the $2 \mathrm{~N}$ and $3 \mathrm{~N}$ calculations correspond to $\Delta(a b s(3 N-2 N) \geq 0.03$.

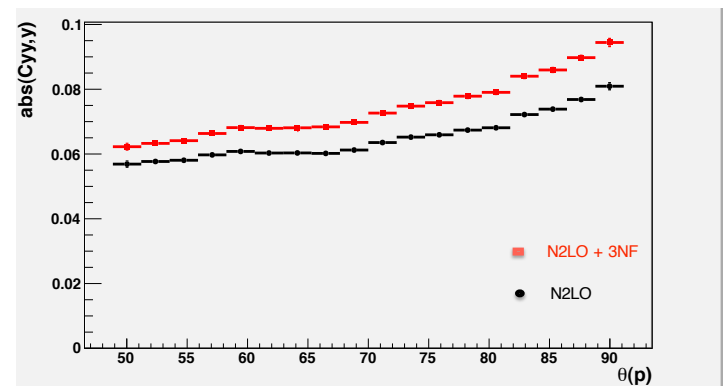

Figure 6: The absolute magnitude of the tensorvector correlation coefficient $C_{y y, y}$ as function of $\theta_{p}$ in a profile histogram using conditions on $p, \theta_{p}, \phi_{p}$ and $\phi_{q}$.

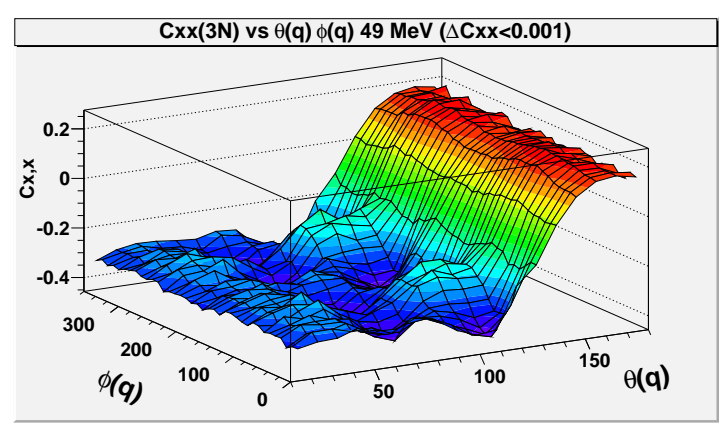

Figure 8: The vector-vector correlation coefficient $C_{x, x}(3 \mathrm{~N})$ as function of $\theta_{q}$ and $\phi_{q}$ at $49 \mathrm{MeV}$ with a condition applied on the absolute difference between the $2 \mathrm{~N}$ and $3 \mathrm{~N}$ theoretical predictions; $\Delta(a b s(3 N-2 N) \leq 00.1$. From this graph one of the conditions is deduced in order to find areas in phase space where the theory predicts similar behavior for $2 \mathrm{~N}$ and $3 \mathrm{~N}$ calculations

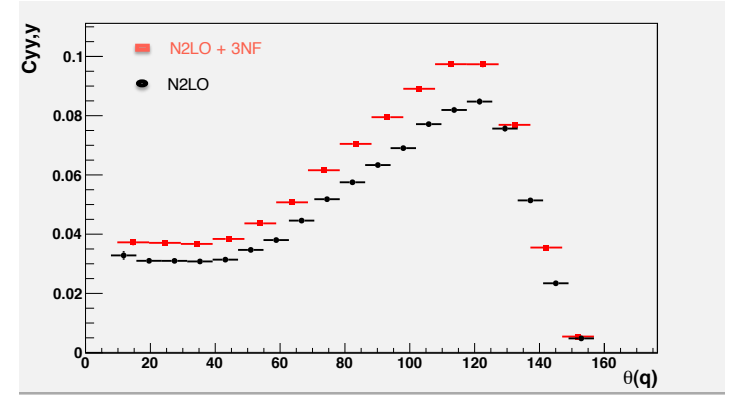

Figure 7: The tensor-vector correlation coefficient $C_{y y, y}$ as function of $\theta_{q}$ in a profile histogram using conditions on $p, \theta_{p}, \phi_{p}$ and $\phi_{q}$, yielding a deviation of roughly 0.02 at 120 degrees.

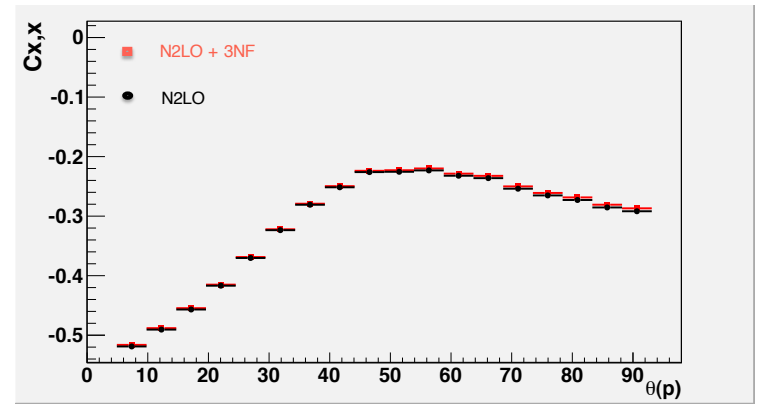

Figure 9: The vector-vector correlation coefficient $C_{x, x}$ predicted from $3 \mathrm{~N}$ (squares, red online) and $2 \mathrm{~N}$ (dots, black online) calculations as function of $\theta_{p}$ in a profile histogram integrated over all other parameters except for selected cuts. In this graph aimed at showing no or insignificant $3 \mathrm{NF}$ effects for a consistent description, the cuts were made on $\theta_{q} \leq 100^{\circ}, q \geq 60$ and $p$ in $[30,130] \mathrm{MeV} / \mathrm{c}$. 


\section{References}

[1] B.v. Przewoski et al., Analyzing Powers and Spin Correlation Coefficients for p $+d$ Elastic Scattering at 135 and $200 \mathrm{MeV}$, Phys. Rev. C 74064003 (2006) [nucl-ex/ 0411019 ].

[2] K. Sekiguchi et al., Polarization Transfer Measurement for ${ }^{1} H(\vec{d}, \vec{p})^{2} H$ Elastic Scattering at 135 MeV/u and Three Nucleon Force Effects, Phys. Rev. C 70, 014001 (2004) [nucl-ex/ 0404026 v1].

[3] H. O . Meyer et al., Axial Observables in dp Breakup and the Three-Nucleon Force, Phys.Rev. Lett. 93, 112502 (2004).

[4] S. Kistryn et al., Systematic study of three-nucleon force effects in the cross section of the deuteron-proton breakup at 130 MeV, Phys. Rev. C 72, 044006 (2005) [nucl-ex/0508012].

[5] E. Stephan et al., Vector and tensor analyzing powers of elastic deuteron-proton scattering at 130 MeV deuteron beam energy, Phys. Rev. C 76, 057001 (2007).

[6] E. Stephan et al., Vector and tensor analyzing powers in deuteron-proton breakup at $130 \mathrm{MeV}$, Phys. Rev. C 82, 014003 (2010).

[7] N. Kalantar-Nayestanaki, E. Epelbaum, J.G. Messchendorp and A. Nogga, Signatures of three-nucleon interactions in few-nucleon systems, accepted for publication in Rep. Prog. Phys, [nucl-ex/nucl-th/1108.1227].

[8] E. Epelbaum, H.-W. Hammer and Ulf-G. Meißner, Modern theory of nuclear forces, Rev. Mod. Phys. 81 1773-1825 (2009) [nucl-th/ 0811.1338 ].

[9] R. Machleidt, D. R. Entem, Chiral effective field theory and nuclear forces, Phys. Rept. 503, 1 (2011) [nucl-th/1105.2919v1].

[10] D. R. Entem and R. Machleidt, Phys. Rev. C 68, 041001(R) (2003).

[11] E. Epelbaum, W. Glöckle, and Ulf-G. Meißner, Nucl. Phys. A747, 362 (2005).

[12] V. Bernard, E. Epelbaum, H. Krebs and Ulf-G. Meißner, Subleading contributions to the chiral three-nucleon force: Long-range terms, Phys. Rev. C 77, 064004 (2008) [nucl-th/0712.1967v1].

[13] V. Bernard, E. Epelbaum, H. Krebs and Ulf-G. Meißner, Subleading contributions to the chiral three-nucleon force II: short-range terms and relativistic corrections, [nucl-th/1108.3816].

[14] E. Epelbaum, private communication.

[15] D. Oellers and C. Weidemann et al., Spin-Filtering Studies at COSY, update and beam request, COSY Proposal no. 199.2 (2011), submitted to the COSY Program Advisory Committee by the PAX collaboration, available from www2.fz-juelich.de/ikp/pax/portal/index.php?id=404.

[16] P. Lenisa and F. Rathmann et al., Measurement of the Spin-Dependence of the $\bar{p} p$ Interaction at the AD-Ring; AD Proposal SPSC-P-337 (2009), submitted to the AD Program Advisory Committee by the PAX collaboration, available from www2.fz-juelich.de/ikp/pax/portal/index.php?id=404.

[17] P. Thörngren Engblom et al., Measurement of Spin Observables in the $\vec{p} \vec{d}$ Breakup Reaction COSY Proposal 202.1 (2011), submitted to the COSY Program Advisory Committee by the PAX collaboration, www2.fz-juelich.de/ikp/publications/PAC39/PAX_proposal202.1_202.pdf. 
[18] G. G. Ohlsen, Rep. Prog. Phys. 35, 717-801 (1972)

[19] P. Thörngren Engblom et al., New experimental approach to modern three-nucleon forces, 19th International Spin Physics Symposium (SPIN2010) 27 SeptemberĐ2 October, 2010, J§lich, Germany, Journal of Physics: Conference Series 295012118 (2011),

[http://iopscience.iop.org/1742-6596/295/1/012118]

[20] J. Kuros-Zolnierczuk, P. Thörngren Engblom, H.O. Meyer, T.J. Whitaker, H. Witala, J. Golak, H. Kamada, A. Nogga, R. Skibinksi, Faddeev calculations of break-up reactions with realistic experimental constraints, Few Body Syst. 34, 259-273 (2004) [nucl-th/ 0402030 ].

[21] P. Thörngren Engblom et al., Experimental search for evidence of the three-nucleon force and a new analysis method, The 19th European Few-Body Conference, Groningen Aug. 23-27, 2004, Few-Body Problems in Physics, eds. N. Kalantar-Nayestanaki, R.G.E. Timmermans and B.L.G. Bakker, AIP Conf. Proc. 768, 65-68 (2005) [nucl-ex/ 0410006 ]. 